\title{
Small quark stars in the chromodielectric model
}

\author{
M. Malheiro*, E.O. Azevedo*, L.G. Nuss*, M. Fiolhais ${ }^{\dagger}$ and A. \\ Taurines** \\ *Instituto de Física, Universidade Federal Fluminense, Av. Litorânea, 24210-310 Niterói, \\ Brazil \\ ${ }^{\dagger}$ Departamento de Física and Centro de Física Computacional, Universidade de Coimbra, \\ P-3004-516 Coimbra, Portugal \\ ** Instituto de Física, Universidade Federal do Rio Grande do Sul CP 15051, 91501-970 \\ Porto Alegre, Brazil
}

\begin{abstract}
Equations of state for strange quark matter in beta equilibrium at high densities are used to investigate the structure (mass and radius) of compact objects. The chromodielectric model is used as a general framework for the quark interactions, which are mediated by chiral mesons, $\sigma$ and $\vec{\pi}$, and by a confining chiral singlet dynamical field, $\chi$. Using a quartic potential for $\chi$, two equations of state for the same set of model parameters are obtained, one with a minimum at around the nuclear matter density $\rho_{0}$ and the other at $\rho \sim 5 \rho_{0}$. Using the latter equation of state in the Tolman-Oppenheimer-Volkoff equations we found solutions corresponding to compact objects with $R \sim 5-8 \mathrm{~km}$ and $M \sim M_{\odot}$. The phenomenology of recently discovered X-ray sources is compatible with the type of quark stars that we have obtained.
\end{abstract}

\section{INTRODUCTION}

Various effective models using quarks as fundamental dynamical fields, originally designed for the nucleon, have also been successfully used to describe infinite quark matter, and the resulting equations of state (EOS's) have been applied to investigate the structure of compact stars [1-6].

The chromodielectric model (CDM) [7-9], for example, provides a reasonable phenomenology for the nucleon $[10,11]$ and also allows us to obtain EOS's for dense quark matter. The model yields soliton solutions representing single baryons with three quarks dynamically confined by a scalar field, $\chi$, whose quanta can be assigned to $0^{++}$glueballs. When it is applied to quark matter in two or three flavors [12-14] the resulting EOS turns out to be relatively soft at large densities.

Using a quadratic potential for the $\chi$ field, Drago et al. $[15,16]$ applied the CDM to describe the inner part of neutron stars, obtaining masses in the range $1-2 M_{\odot}$ and radii of the order $10 \mathrm{~km}$ or higher, with a small hadron crust of $2 \mathrm{~km}$. In this work we consider an extension of the model used in Ref. [15], taking quartic instead of quadratic potentials. In addition to the structures found by Drago et al., the quartic model predicts another type of compact objects made out of quarks only, smaller and denser than neutron stars. 
From the observational point of view, the recent discovery of X-ray sources, by the Hubble and Chandra telescopes, increased the plausibility that these sources might be strange quark stars [17-19]. In particular, the isolated compact object RX J1856.5-3574 with a small radius does not show evidence of spectral lines or edge features $[20,21]$, reinforcing the conjecture for the existence of stars made out of strange matter. The phenomenology of these objects seems to be compatible with the small and dense quark stars reported in this work.

\section{THE MODEL}

\section{CDM Lagrangian}

We write the CDM Lagrangian in the form [8-10]

$$
\mathcal{L}=\mathcal{L}_{q}+\mathcal{L}_{\sigma, \pi}+\mathcal{L}_{q-\text { meson }}+\mathcal{L}_{\chi}
$$

where

$$
\mathcal{L}_{q}=\mathrm{i} \bar{\psi} \gamma^{\mu} \partial_{\mu} \psi, \quad \mathcal{L}_{\sigma, \pi}=\frac{1}{2} \partial_{\mu} \hat{\sigma} \partial^{\mu} \hat{\sigma}+\frac{1}{2} \partial_{\mu} \hat{\vec{\pi}} \cdot \partial^{\mu} \hat{\vec{\pi}}-W(\hat{\vec{\pi}}, \hat{\sigma}),
$$

and $W(\hat{\vec{\pi}}, \hat{\sigma})$ is the Mexican hat potential. In the $u, d$ sector the quark-meson interaction is described by

$$
\mathcal{L}_{q-\text { meson }}=\frac{g}{\chi} \bar{\psi}\left(\hat{\sigma}+\mathrm{i} \vec{\tau} \cdot \hat{\vec{\pi}} \gamma_{5}\right) \psi .
$$

The last term in (1) contains the kinetic and the potential piece for the $\chi$-field:

$$
\mathcal{L}_{\chi}=\frac{1}{2} \partial_{\mu} \hat{\chi} \partial^{\mu} \hat{\chi}-U(\hat{\chi})
$$

The potential term is

$$
U(\chi)=\frac{1}{2} M^{2} \chi^{2}\left[1+\left(\frac{8 \eta^{4}}{\gamma^{2}}-2\right) \frac{\chi}{\gamma M}+\left(1-\frac{6 \eta^{4}}{\gamma^{2}}\right) \frac{\chi^{2}}{(\gamma M)^{2}}\right],
$$

where $M$ is the $\chi$ mass. The parameterization used in (5) allows for a physically meaningful interpretation of the parameters $\gamma$ and $\eta$ : $U(\chi)$ has a global minimum at $\chi=0$ and a local one at $\chi=\gamma M$, and $U(\gamma M)=\eta^{4} M^{4}$ (see Fig. 1). The height of the local minimum, $B=(\eta M)^{4}$, is interpreted as a "bag pressure" and this is used to fix the parameters in $U(\chi)$. Assuming the wide range $0.150 \leq B^{1 / 4} \leq 0.250$ $\mathrm{GeV}$, one has $0.08 \leq \eta \leq 0.15$, using $M=1.7 \mathrm{GeV}$. We note that $\gamma$ is not a free parameter since the quartic term of $U(\chi)$ must be positive and the cubic term negative, which implies $\gamma^{2} \geq 6 \eta^{4}$. In the soliton sector of the model, best nucleon properties are obtained for $G=\sqrt{g M} \sim 0.2 \mathrm{GeV}$ (only $G$ matters for the nucleon properties) and we keep that $G$ in our quark matter calculations.

In order to study strange quark matter, we add to the interaction Lagrangian (3) the term [22]

$$
\mathcal{L}_{s-\text { meson }}=\frac{g_{s}}{\chi} \bar{\psi}_{s} \psi_{s}
$$

accounting for the coupling between the strange quark and the $\chi$ field. 


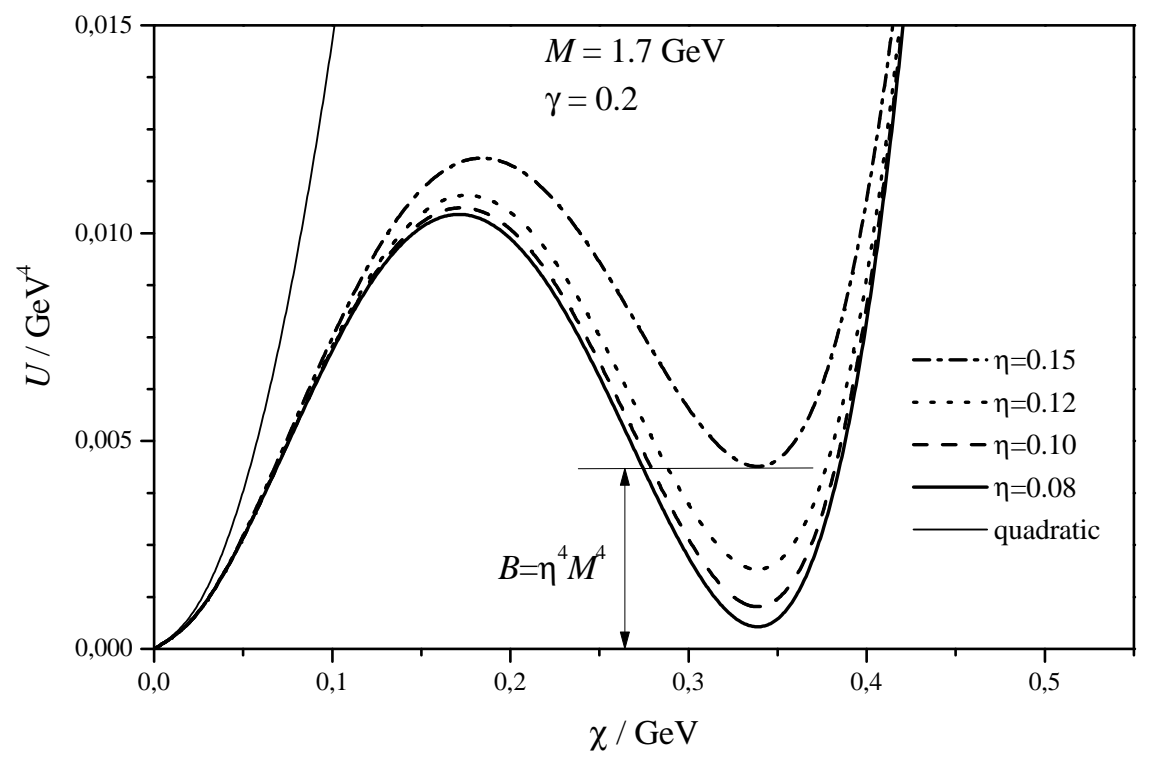

FIGURE 1. Quartic potential, Eq. (5), for fixed $M$ and $\gamma$, in dependence of $\chi$. For comparison, the quadratic potential $(\gamma \rightarrow \infty)$ is also shown. The value of the potential at the second minimum can be interpreted as a bag pressure, $B$.

\section{Strange quark matter}

In order to study strange quark matter in beta equilibrium, an electron gas must also be considered. The mean-field energy per unit volume for strange quark matter in the CDM (plus electrons) is given by

$$
\begin{aligned}
\varepsilon & =\alpha \sum_{f=u, d} \int_{0}^{k_{f}} \frac{d^{3} k}{(2 \pi)^{3}} \sqrt{k^{2}+m_{f}(\sigma, \chi)^{2}}+\alpha \int_{0}^{k_{s}} \frac{d^{3} k}{(2 \pi)^{3}} \sqrt{k^{2}+m_{s}(\chi)^{2}} \\
& +2 \int_{0}^{k_{e}} \frac{d^{3} k}{(2 \pi)^{3}} \sqrt{k^{2}+m_{e}^{2}}+U(\chi)+\frac{m_{\sigma}^{2}}{8 f_{\pi}^{2}}\left(\sigma^{2}-f_{\pi}^{2}\right)^{2},
\end{aligned}
$$

where the first two terms refer to quarks and the third one to the electrons, all described by plane waves. The degeneracy factor is $\alpha=6$ (for spin and color). The last term is the Mexican hat potential (with $\vec{\pi}=0$ and $f_{\pi}=93 \mathrm{MeV}$ ). The $k_{i}$ in (7) are the Fermi momenta of quarks and electrons.

The quark masses in (7) are [22]: $m_{u, d}=g_{u, d} \sigma /\left(\chi f_{\pi}\right)$ and $m_{s}=g_{s} / \chi$ with the coupling constants given by $g_{u}=g\left(f_{\pi}+\xi_{3}\right), g_{d}=g\left(f_{\pi}-\xi_{3}\right)$ and $g_{s}=g\left(2 f_{k}-f_{\pi}\right)$ $\left[\xi_{3}=-0.75 \mathrm{MeV}, f_{K}=113 \mathrm{MeV}\right]$.

A variational principle applied to the energy density, Eq. (7), leads to two gap equations for $\sigma$ and $\chi$. In the interior of a compact star the matter should satisfy both the electrical charge neutrality and chemical equilibrium. These conditions should supplement the gap equations, and altogether we have a system of six 
algebraic equations to solve at each baryon density $\rho=\left(\rho_{u}+\rho_{d}+\rho_{s}\right) / 3$ [here, $\rho_{i}=$ $\alpha k_{i}^{3} /(6 \pi)^{2}$ stand for each flavor density]. The solution of the system of equations are the meson fields, $\sigma$ and $\chi$, and the Fermi momenta, $k_{u}, k_{d}, k_{s}$ and $k_{e}$. For the same set of model parameters we found two stable solutions, hereafter denoted by I and II, shown in Fig. 2.
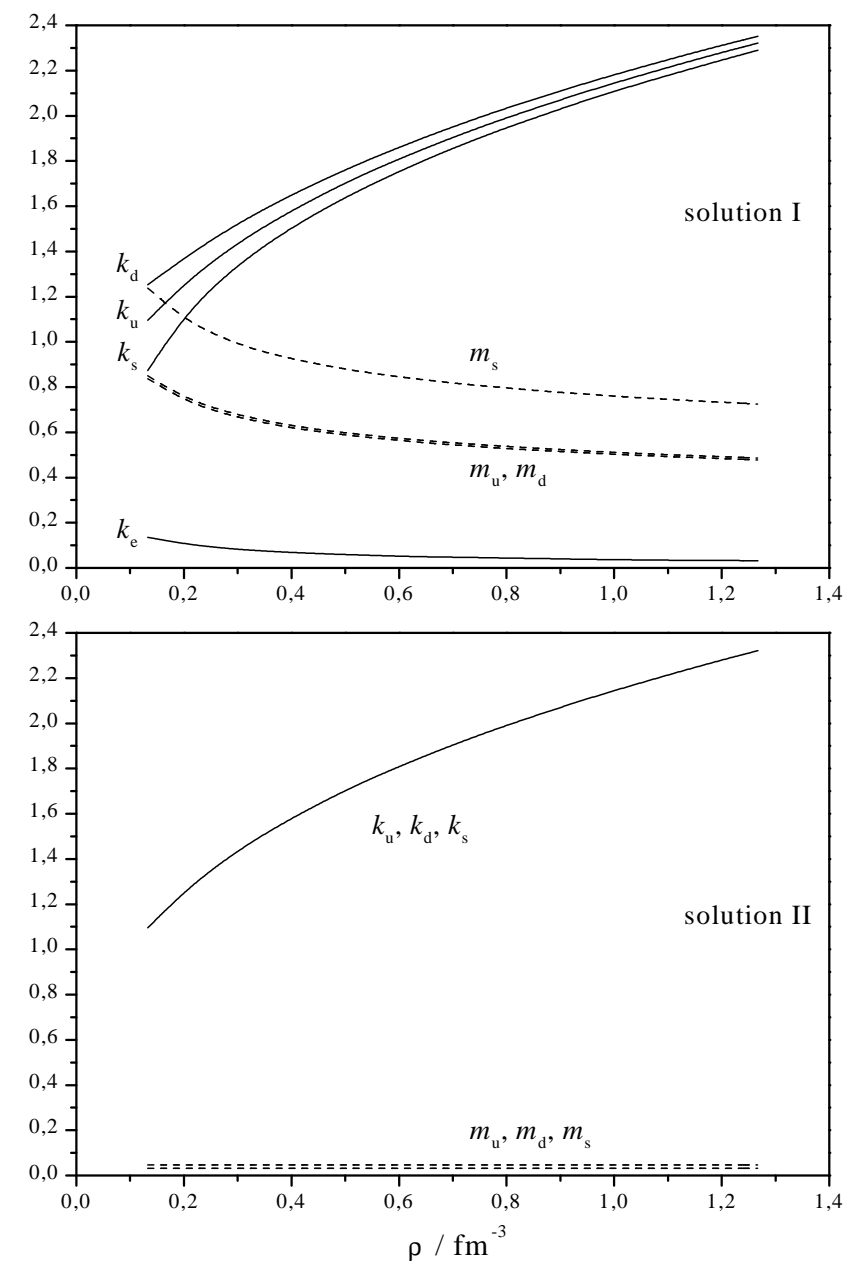

FIGURE 2. Fermi momenta (solid lines) and quark masses (dashed lines) for solution I and II in dependence of the baryon density. For solution II the quark masses and the electron Fermi momentum almost vanish. The model parameters are $g=0.023 \mathrm{GeV}, M=1.7 \mathrm{GeV}, \gamma=0.2$ and $\eta=0.12$. The vertical scales are in $\mathrm{fm}^{-1}$.

For both solutions $\sigma$ is always close to $f_{\pi}$. In solution I, the $\chi$ field is a slowly increasing function of the density, remaining always smaller than $\sim 0.05 \mathrm{GeV}$. For such a small $\chi$, the quartic potential and the quadratic potential are indistinguishable (see Fig. 1), thus, in practice, solution I corresponds to the one obtained and used by Drago et al. [15] in the framework of the quadratic potential. Due to the smallness of the $\chi$ field, quark masses are large and the system is in a chiral broken phase. The solution II exhibits a large confining field, $\chi \sim \gamma M$ (local minimum of $U$ ), independent of the density. The resulting quark masses are similar for the 
three flavors and very close to zero (chiral restored phase). Therefore, the chemical potentials in solution II are dominated by the Fermi momentum contribution, $\mu_{u} \simeq \mu_{d} \simeq \mu_{s}$ and $\mu_{e} \simeq 0$, i.e. in solution II there are almost no electrons. Besides solutions I and II, there is an additional unstable solution corresponding to $\chi \sim \gamma M / 2$ [local maximum of $U(\chi)$ ].

\section{Equations of state}

The energy per baryon number as a function of the baryon density (EOS) is readily evaluated for each solution. We stress that EOS-I is not sensitive to $\gamma$ and $\eta$ (since $\chi$ is small), just depends on $G$. The saturation density occurs at a relatively low density $\left(\rho=1.2-1.6 \rho_{0}\right)$ and the behavior of EOS-I at intermediate densities, is similar to the hadronic EOS's (see Ref. [13] for the two flavors sector). The EOS-II is also insensitive to $\gamma$, but does depend on $\eta$ [in fact, the dependence is on $(\eta M)^{4}$, as we have already pointed out]: the energy per baryon number increases with $\eta$ and so does the saturation density. For $\eta \sim 0.12$ the saturation density is $\rho \sim 5 \rho_{0}$ and the energy per baryon number is some $20 \%$ higher than for solution I at its saturation density.
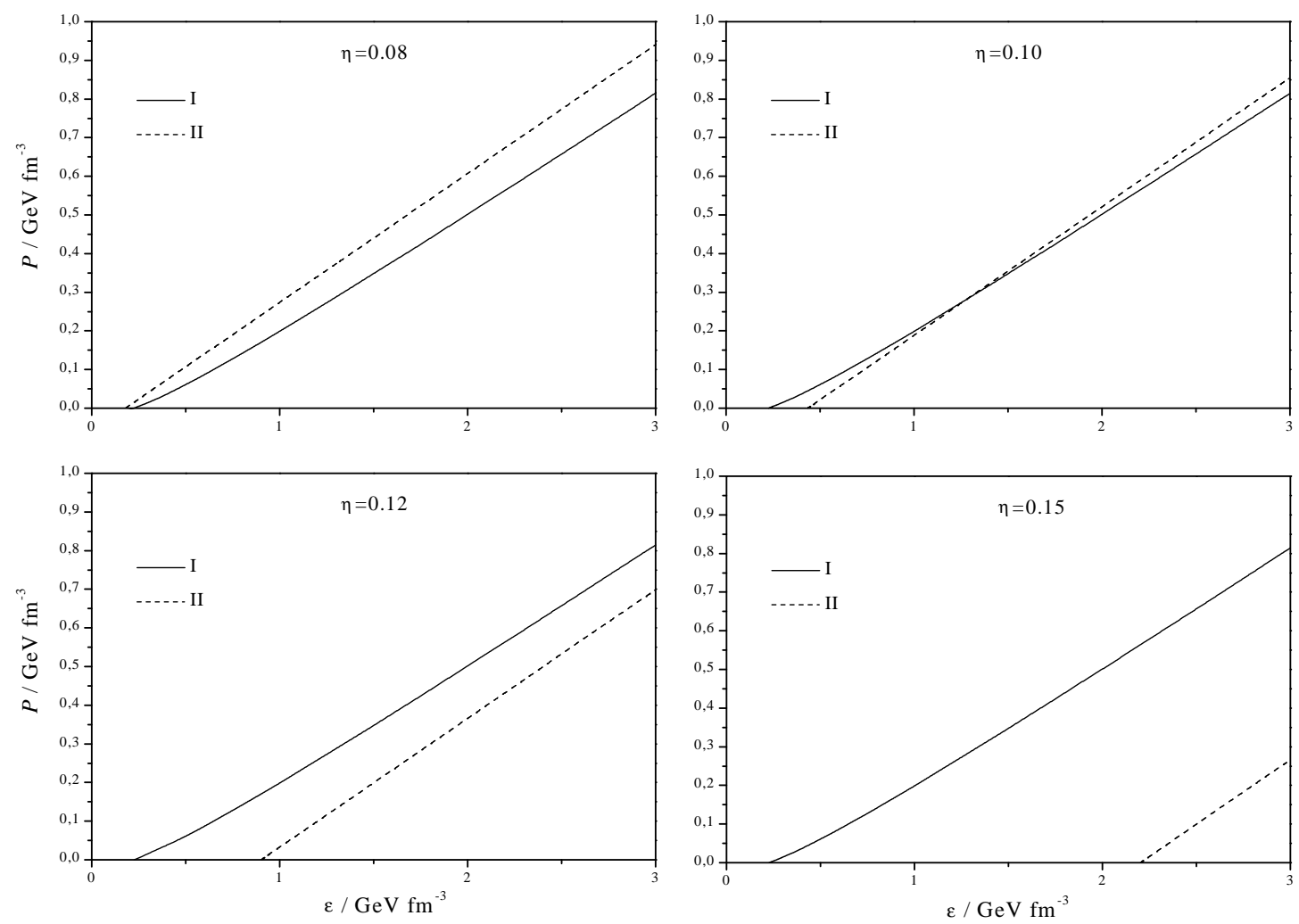

FIGURE 3. Pressure versus energy density for the two types of solution for various parameters $\eta$. ] The other model parameters are $M=1.7 \mathrm{GeV}, g=0.023 \mathrm{GeV}$ and $\gamma=0.2$. 
In Fig. 3 we present the EOS's in the form of pressure $P=\rho^{2} \frac{\partial}{\partial \rho}\left(\frac{\epsilon}{\rho}\right)$ versus energy density plots for solutions I and II and various $\eta$. It is worth noticing that our results with two distinct EOS predicted by the CDM, are consistent with the results from perturbative QCD: it is remarkable indeed that, for $\eta \sim 0.12$ the CDM reproduces accurately the EOS's recently obtained in a perturbative QCD calculation (compare our third panel of Fig. 3 with figure 1 of Ref. [23]).

Regarding energetics, both phases are almost degenerated at high densities and have similar shapes in the $P \times \epsilon$ plane even at intermediate densities (or energy densities) in the narrow range $0.1 \leq \eta \leq 0.12$. In that region of $\eta$, one solution is not clearly lower in energy than the other. However, we should point out that they correspond to two different $\chi$ values and for the system to undergo a transition from the chiral restored to the chiral broken phase it has to go through a high potential energy barrier. In a 3D plot of the energy per baryon number versus $(\rho, \chi)$ the stable solutions correspond to two distinct "valleys", and the unstable solution mentioned at the end of the previous section corresponds to the top of the barrier between the two valleys.

\section{QUARK STARS}

In order to investigate the structure of stars we solved the Tolman-OppenheimerVolkoff (TOV) equation using the two EOS. Since EOS-I is identical to the one using a quadratic potential, it leads to stars that have the same phenomenology as the hybrid stars obtained by Drago et al. [15]: $R \sim 10-12 \mathrm{~km}$, a hadron crust and a mass $M \sim 1-2 M_{\odot}$. At low densities, hadronization occurs and an hadronic equation of state should be used, replacing EOS-I.

Since EOS-II saturates at a high density and, in addition, the system is not likely to undergo a transition to solution I, one should not perform any connection to the hadronic sector: the EOS-II alone generates a new family of strange quark stars. In Fig. 4 it is shown the mass-radius relation for different values of $\eta$. These quark stars are smaller and denser in comparison with those resulting from EOS-I. For $\eta \sim 0.115$ (and $M=1.7 \mathrm{GeV}$, yielding $B^{1 / 4} \sim 0.195 \mathrm{GeV}$ ) one obtains a maximum radius $R \sim 6 \mathrm{~km}$ and a corresponding mass $M \sim 0.9 M_{\odot}$, which are the fitted radius and mass for the nearby compact object RX J 1856.5-3754 [20, 21]. According to our calculation, such star has a central density of $10 \rho_{0}$ ( $\rho_{0}$ is the nuclear matter density) and a central energy density $\epsilon \sim 3 \times 10^{15} \mathrm{~g} / \mathrm{cm}^{3}$. At the edge, the density drops to $5 \rho_{0}$ and $\epsilon \sim 1.35 \times 10^{15} \mathrm{~g} / \mathrm{cm}^{3}$. The ratio $\epsilon / \rho$ remains approximately constant inside the star. The maximum period of the star, computed using the expression given in Ref. [24], is $\sim 0.4$ ms. From Fig. 4 one concludes that the mass-radius relation for these strange small stars mainly depends on the height of the local minimum of the $\chi$ potential. 


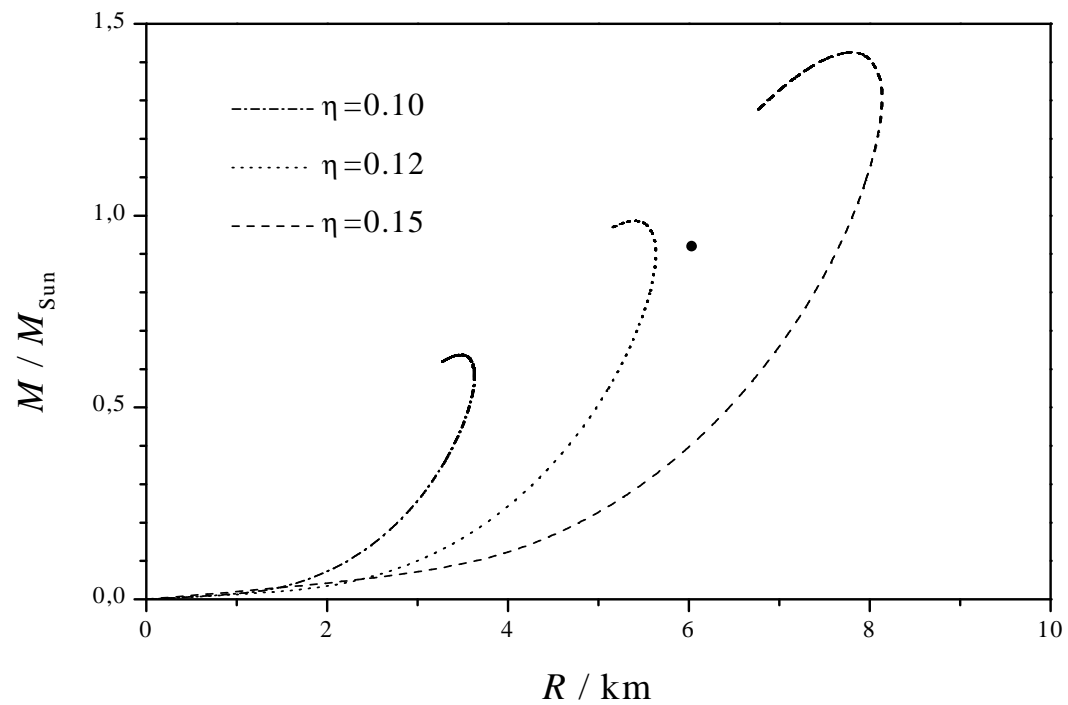

FIGURE 4. Mass versus radius for the pure quark stars (solution II) in the CDM model. The dot indicates the maximum radius star for $\eta=0.115$. For the other model parameters see caption of Fig. 3.

\section{CONCLUSIONS}

Using a mean-field variational method we obtained two solutions for homogeneous strange matter in beta equilibrium using the CDM with a quartic potential, with its parameters fixed in the nucleon sector and to yield a reasonable bag constant.

One solution is similar to the already known solution for quadratic potentials in the CDM, with massive quarks. In the other solution, quarks are almost massless. The EOS for both solutions are similar to those found recently in the framework of perturbative QCD.

The pure quark stars emerging from the chiral symmetric solution are small and dense compact objects, showing a phenomenology compatible with the nearby $\mathrm{X}$-ray source whose mass and radius was recently fitted [20, 21].

\section{ACKNOWLEDGMENTS}

This work was supported by FCT (POCTI/FEDER program), Portugal and by $\mathrm{CNPq} / \mathrm{ICCTI}$ through the Brazilian-Portuguese scientific exchange program. We thank G. Marranghello and B. Garcia for some useful discussions. E.O.A. and L.G.N. acknowledge the support of the PIBIC/CNPq program for young researchers. 


\section{REFERENCES}

1. N. K. Glendenning, Compact Stars - Nuclear Physics, Particle Physics, and General Relativity (Springer, New York, 1997)

2. F. Weber, Pulsars as Astrophysical Laboratories for Nuclear and Particle Physics, (IOP, UK, 1999)

3. H. Heiselberg, M. Hjorth-Jensen, Phys. Rept. 328, 237 (2000)

4. M. Hanauske, L. M. Satarov, I. N. Mishustin, H. Stocker, W. Greiner, Phys. Rev. D 64, 043005 (2001)

5. E. Witten, Phys. Rev. D 30, 272 (1984); C. Alcock, E. Farhi, A. Olinto, Astrophys. J. 310, 261 (1986); P. Haensel, J. L. Zdunik, R. Schaeffer, Astron. Astrophys. 160, 121 (1986)

6. A. R. Taurines, C. A. Z. Vasconcellos, M. Malheiro, M. Chiapparini, Phys. Rev. C 63, 065801 (2001)

7. H. B. Nielsen, A. Patkós, Nucl. Phys. B 195, 137 (1982)

8. H. J. Pirner, Prog. Part. Nucl. Phys., 29, 33 (1992)

9. M. K. Banerjee, Prog. Part. Nucl. Phys., 31, 77 (1993)

10. T. Neuber, M. Fiolhais, K. Goeke, J. N. Urbano, Nucl. Phys. A 560, 909 (1993)

11. A. Drago, M. Fiolhais, U. Tambini, Nucl. Phys. A 609, 488 (1996)

12. S. K. Ghosh, S. C. Phatak, Phys. Rev. C 52, 2195 (1995)

13. A. Drago, M. Fiolhais, U. Tambini, Nucl. Phys. A 588, 801 (1995)

14. W. Broniowski, M. Čibej, M. Kutschera, M. Rosina, Phys. Rev. D 41, 285 (1990)

15. A. Drago, U. Tambini, M. Hjorth-Jensen, Phys. Lett. B 380, 13 (1996); Prog. Part. Nucl. Phys. 36, 407 (1996)

16. A. Drago, A. Lavagno Phys. Lett. B 511, 229 (2001)

17. I. Bombaci, A. V. Thampan, B. Datta, Astrophys. J. 541, L71 (2000)

18. X. D. Li, I. Bombaci, M. Dey, J. Dey, E. P. J. van den Heuvel, Phys. Rev. Lett. 83, 3776 (1999)

19. M. Dey, I. Bombaci, J. Dey, S. Ray, B. C. Samanta, Phys. Lett. B 438, 123 (1998)

20. J. A. Pons, F. M. Walter, J. M. Lattimer, M. Prakash, R. Neuhäuser, P. An, Astrophys. J. $\mathbf{5 6 4}, 981(2002)$

21. J. J. Drake et al., astro-ph/0204159

22. J. A. McGovern, M. Birse, Nucl. Phys. A 506, 367 (1990); Nucl. Phys. 506, 392 (1990)

23. E. S. Fraga, R. D. Pisarski, J. Schaffner-Bielich, Phys. Rev. D 63, 121702 (2001)

24. P. Haensel, J. L. Zdunik, Nature 340617 (1989) 